\title{
INSPIRE: An INtelligent System for Personalized Instruction in a Remote Environment
}

\author{
Kyparisia A. Papanikolaou ${ }^{1}$, Maria Grigoriadou ${ }^{1}$, Harry Kornilakis ${ }^{1}$, and \\ George D. Magoulas ${ }^{2}$ \\ 1 Department of Informatics \& Telecommunications \\ University of Athens, Panepistimiopolis \\ GR-15784 Athens, Greece \\ \{spap, gregor, harryk\}@di.uoa.gr \\ 2 Department of Information Systems and Computing \\ Brunel University \\ West London, UB8 3PH \\ George.Magoulas@brunel.ac.uk
}

\begin{abstract}
In this paper we present the architecture of an Adaptive Educational Hypermedia System, named INSPIRE. This particular system, throughout its interaction with the learner, dynamically generates lessons that gradually lead to the accomplishment of the learning goals selected by the learner. The lessons are generated according to the learner's knowledge level, learning style and follow his/her progress. The adaptive behavior of the system, the functionality of the various modules and the opportunities offered to learners for intervention are presented.
\end{abstract}

\section{Introduction}

Adaptive Educational Hypermedia Systems (AEHS) 32, extend the benefits derived from the instructional use of the Web by incorporating the idea of offering learners personalized support and/or instruction in a distance learning setting. The adaptive characteristics of an Educational Hypermedia System usually aim to both usability and learning. Thus, the educational implications are very important and should be considered during the design and development stages of the system. Although many questions are still open in the area of Instructional Design about instruction / learning and how it is efficiently provided / attained [17, it is important to consider adaptation within the framework of current learning theories and models, and thoroughly plan the sharing of the task of adaptation between the learner and the system.

We have developed an AEHS, named INSPIRE. Based on the learning goal that the learner selects, INSPIRE generates lessons that correspond to specific learning outcomes accommodating learner's knowledge level and learning style. Thus, aiming at individualizing instruction, the system generates lesson plans tailored to the needs, preferences and knowledge level of each individual learner 
by making use of information about the learner gathered through their interaction. Furthermore, the system provides learners with the option to intervene, expressing their perspective about their own characteristics, or about the lesson contents, and accordingly formulate their interaction with the system, in an attempt to engage learners in the learning process.

\section{INSPIRE's Adaptive Functionality}

The proposed system aims to facilitate distance learners during their study, adopting a pedagogical framework inspired by theories of the area of Instructional Design and Adult Learning. In the beginning of the interaction, the domain knowledge presented to the learner is restricted and gradually it is enriched, following the internal structure of the domain (curriculum sequencing technique), while a navigation route is pro-posed based on learner's progress (adaptive navigation technique).

The main instructional outcomes of the generated lessons on learners' level of performance are to: understand and remember the most important instances and generalities associated with the learning goal they study (Remember); be able to apply them to specific cases (Use); and be able to generate new generalities (Find) [13. The presentation of the educational material provided for each different level of performance, i.e. Remember, Use and Find, is mainly determined by the learning style of the learner (adaptive presentation technique). Thus, learners' preferences that usually guide systems' adaptation [3], are determined based on their learning style. Following the theory of learning styles [4, 912 , how much individuals learn, i.e. the effectiveness of instructional manipulations, is mainly influenced by the educational experiences geared toward their particular style of learning. This approach to learning emphasizes the fact that individuals perceive and process information in very different ways. In this paper we propose a framework for the system's adaptive behavior that exploits the information of the learning style. The learning style model that we adopted in the current implementation of the system is that of [8], where Honey and Mumford, based on Kolb's theory of experiential learning [9], suggested four types of learners: Activists, Pragmatists, Reflectors and Theorists.

The proposed system also supports end-learner modifiability offering opportunities to the learners to intervene in different stages of the lesson generation process, as well as on the construction of their learner model. Thus, learners have the option to activate or deactivate the lesson generation process of the system. In case of activation, they have the option to guide system's instructional decisions by updating accordingly their characteristics on their model, i.e. their knowledge level on the different concepts of the learning goal and their learning style. The externalization of the model to the learners is implemented in a manner that allows it to be understandable, transferable and usable [7]. 


\section{The Architecture of INSPIRE}

INSPIRE's architecture has been designed so as to facilitate knowledge communication between the learner and the system and support its adaptive functionality. INSPIRE is comprised of five different modules (see Fig. 1): (i) the Interaction Monitoring Module that monitors and handles learner's responses during his/her interaction with the system, (ii) the Learner's Diagnostic Module, which processes data recorded about the learner and decides on how to classify the learner's knowledge, (iii) the Lesson Generation Module that generates the lesson contents according to learner's knowledge goals and knowledge level, (iv) the Presentation Module that generates the educational material pages sent to the learner, and (v) the Data Storage, which holds the Domain knowledge and the Learner's Model.

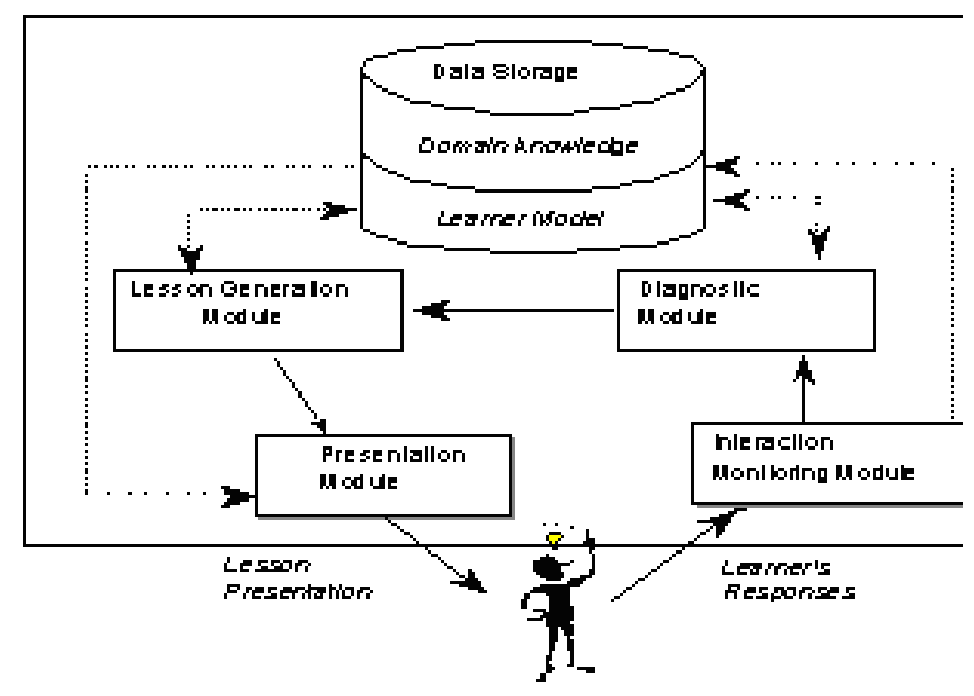

Fig. 1. INSPIRE's components and the interactions with the learner

\subsection{Representing Knowledge about the Domain and the Learner: Data Storage}

The domain knowledge of the system is structured in three hierarchical levels of knowledge abstraction: learning goals, concepts and educational material [15]. Every learning goal is associated with a subset of concepts. Assigning qualitative characterizations provides interrelation among the different concepts of a learning goal, i.e. outcome concepts, prerequisite concepts and related concepts. Note that the prerequisites and related concepts are linked to specific outcome 
concepts. The outcome concepts of a learning goal are further organized in a layered structure, i.e. the outcome concepts of the ith layer should be presented before the outcome concepts of the next, $\mathrm{i}+1$, layer.

The educational material related to each outcome concept consists of knowledge modules, developed according to three levels of performance: Remember, Use and Find, as proposed in [13. Each level of performance is associated with a different combination of multiple types of knowledge modules aiming at increasing the learning efficiency: (i) the Remember level of performance includes information necessary to present the concept, i.e. expository and inquisitory theory presentations and/or examples plus images and/or questions and self-estimation tests, assessment tests, (ii) the Use level of performance includes information necessary to apply the concept to specific cases, i.e. hints from the theory and/or examples and/or exercises and/or activities based on computer simulations, selfestimation tests, assessment tests, and (iii) the Find level of performance aims to the ability of the learner to find a new concept, principle, procedure, and thus the educational material provided includes activities on simulations, exploration activities, case studies. The representation of the educational material pages in the database is based on the ARIADNE recommendation for educational metadata [1]. Metadata specify the attributes that fully and adequately describe the knowledge modules of the educational material.

The learner model. The learner model is the system's representation of the learner. It supports learner's communication with the system and reflects some of his/her features. It describes the learner (general information, learning style) and his/her "current state" (knowledge level on the different concepts and learning goals, performance on assessment tests, number, type and order of resources $\mathrm{s} /$ he has accessed etc.).

The knowledge level of the learner on a certain concept is assigned one of the characterizations I, RS, AS, S = Insufficient, Rather Sufficient, Almost Sufficient, Sufficient. This assignment is made based on learners' answers to assessment questions of different types. The diagnostic module uses the approach described in [10] for multicriterial decision-making in order to assess learner's knowledge level on each particular concept of a learning goal.

Currently, the dominant learning style of the learner, which is stored in his/her profile, is initialized by means of a Honey \& Mumford questionnaire 8, which the learner fills in the first time s/he logs on the system. Alternatively, the learner is provided with the option to directly select his/her dominant learning style, based on information provided by the system about the general characteristics of the different learning style categories.

\subsection{Monitoring the Learner: Interaction Monitoring Module}

The function of the Interaction-Monitoring Module is to log the requests made by the learner, as part of his/her HTTP request, and update the learner's model with the newly acquired information. Since the interaction-monitoring module is the only part of INSPIRE that receives direct input from the learner, it is responsible for collecting data concerning the learners' observable behavior and 
for notifying the other modules about their actions; examples of such actions are: the inspection or modification of his/her model, the selection of a learning goal and the activation/deactivation of the lesson generation process.

\subsection{Planning the Lesson's Contents: Lesson Generation Module}

The Lesson Generation Module realizes the lesson generation process, which plans the content and the delivery of each lesson. The outcome concepts of a learning goal are presented gradually according to the priority of the layer they belong to. The lesson generation process determines which one of the layers of the outcome concepts (as described in Sect. 3.1 about the structure of the domain knowledge) should be proposed to the learner (see in Fig.4 the lesson contents in the Navigational Area). This decision is mainly guided by learner's knowledge level on the outcome concepts of the previous layers.

Every outcome concept of the selected layer is accompanied by its prerequisites and related ones. In the proposed approach we use different strategies for planning the content of a lesson in each particular layer. This process takes into account the relative importance of each concept on the learning goal as well as the knowledge level of the learner on those concepts. For example:

1. If the knowledge level of the learner has been evaluated as \{Insufficient\} on a number of outcome concepts. Then, s/he has to study the educational material of the Remember level on these outcome concepts and their entire prerequisite ones.

2. If the knowledge level of the learner has been evaluated as \{Rather Sufficient $\}$ on a number of outcome concepts and \{Sufficient\} on several prerequisite concepts. Then, s/he has to study the educational material of the Use level on these outcome concepts and the rest of the prerequisite ones of the outcome.

The relative importance of the concepts included in a lesson determines the extent of their presentation. Thus, the generated lesson includes: (i) complete presentation of the outcome concepts (according to the three levels of performance), (ii) links to brief presentations of the prerequisite concepts, focusing on their relation to the outcome if necessary and (iii) links to the definition of the related concepts in a glossary. The educational material associated to each of the concepts is predefined, and its presentation to the learner is tailored to his/her learning style. Furthermore, the results of the lesson generation process on the contents and the delivery of the generated lessons are reflected on the navigational route that the system proposes to the learner (this will be described in more detail below).

\subsection{Presenting the Lesson: Presentation Module}

The Presentation Module is responsible for the presentation of the lesson to the learner. After the lesson contents have been specified by the Lesson Generation 
Module using information on the knowledge level of the learner, the Presentation Module takes over to reflect these contents as a navigation route in the domain and to decide on the presentation of the educational material based on information on the learning style of the learner.

Adaptive Presentation. Learners with different learning styles view different presentations of the educational material. The main objective is to support learners, following their preferred way of studying. To this end we exploit the information of their learning style in order to guide decisions on the instructional approach proposed to each individual learner.

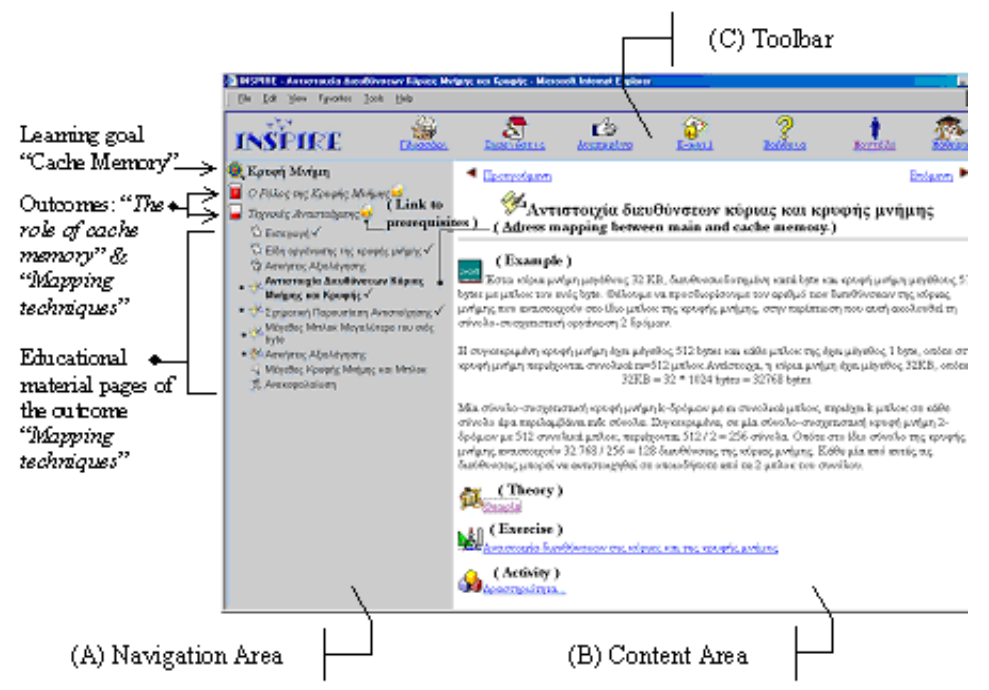

Fig. 2. INSPIRE's main screen presenting the educational material page "Address mapping between main and cache memory" of the outcome concept "Mapping Techniques" of the learning goal "Cache Memory". The screen is divided into three areas: (A) Navigation Area, which includes the contents of the lesson in a hypertext form as links (for clarity purposes the colored icons are denoted by a bullet), (B) Content Area, which presents the contents of the page that the learner selects from the Navigation Area, and (C) Toolbar, which includes several tools that offer learners easy access to various facilities. In the Content Area, different knowledge modules comprising the page of educational material as viewed by Reflectors. Example: description of an application example; Theory: link to hints from the theory; Exercise: link to an exercise; Activity: link to an activity based on a computer simulation.

According to the proposed framework, the multiple external representations of the outcome concepts (expository and inquisitory presentations, examples, exercises, activities based on computer simulations) constitute different instructional primitives [11], which are combined to formulate alternative instructional strategies for the presentation of the educational material. The selection of the 
appropriate instructional strategy for each learning style category reflects some tendencies of the category in approaching information and is in accordance to related work proposed in the literature 616. Furthermore, empirical investigations on the learning preferences of learners have been realized during the first stages of the formative evaluation of INSPIRE aiming at providing direct information about learners attitudes towards the instructional material while studying [14].

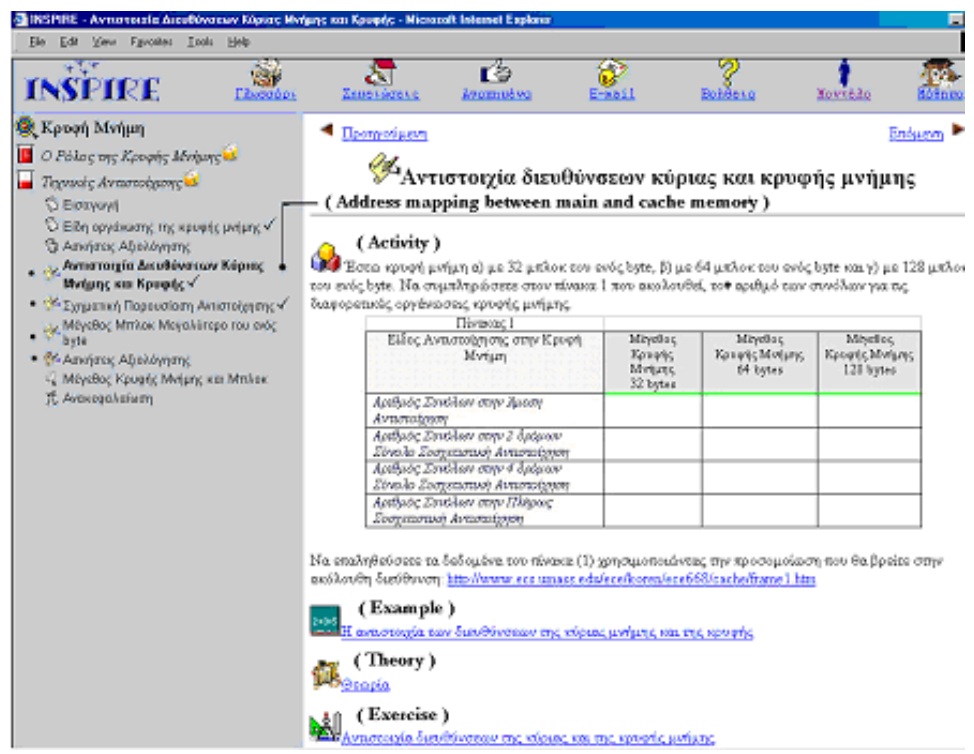

Fig. 3. Different knowledge modules comprising the educational material page "Address mapping between main and cache memory" of the outcome concept "Mapping Techniques" of the learning goal "Cache Memory" as viewed by Activists. Activity: description of an activity in a computer simulation; Example: link to application examples; Theory: link to hints from the theory; and Exercise: link to an exercise.

Although all learners are provided with the same knowledge modules, the method and order of their presentation on each page is adapted, implementing multiple instructional strategies that focus on different perspectives of the concepts. This way, we attempt to maximize the benefit gained from style awareness [89]. Learners are motivated to pass through all the provided educational material exploiting their own capabilities and developing new ones. Consequently, for Reflectors who tend to collect and analyze data before taking action, an example-oriented strategy (see Fig.2) proposes him/her to start reading the example, continue with a brief theory presentation and then try to solve an exercise. Accordingly, for the presentation of material to Activists, who are more motivated by experimentation and attracted by challenge, the instructional strategy adopted is activity-oriented (see Fig.3) and suggests to him/her 
to start experimenting with an activity designed for a computer simulation and provides him/her with the necessary information (example \& theory).

Thus, with regards to the implementation of the adopted instructional strategy, if it is example-oriented then the knowledge module "Example" will be presented on the top of the page while the rest of the modules will appear next as links in a specified order (see Fig. 2); if the instructional strategy is activityoriented then the knowledge module "Activity" will be presented on the top of the page while the rest of the modules will appear next as links in a specified order (see Fig. 3).

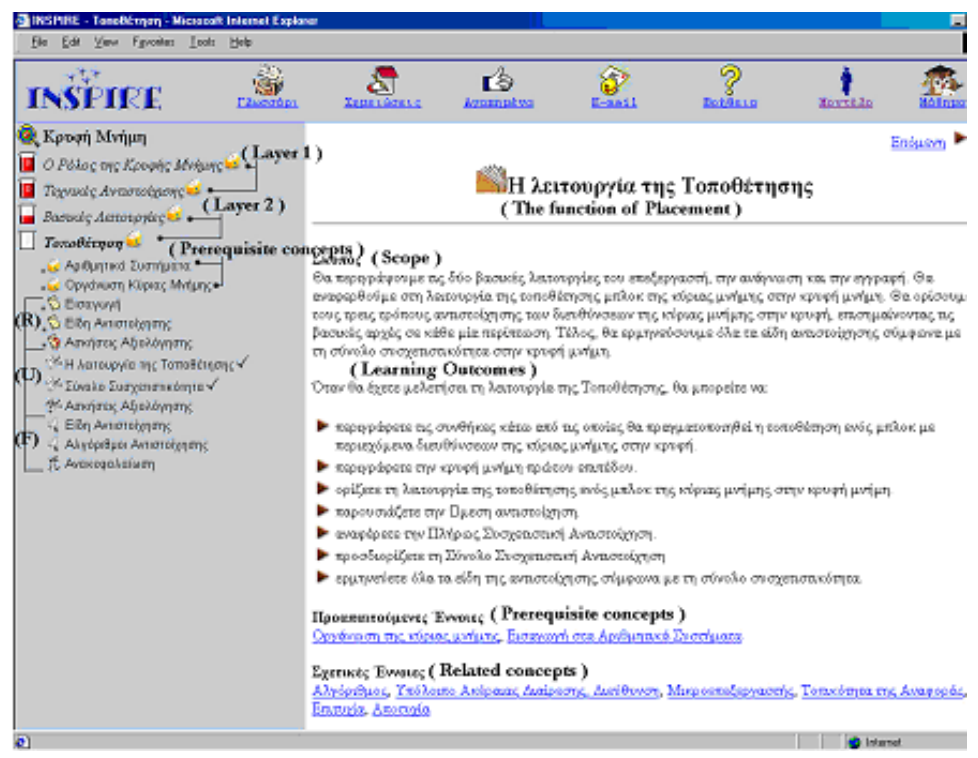

Fig. 4. INSPIRE's main screen presenting the initial page of the outcome concept "Placement". In the Navigation Area note that: the coloured icons are denoted by bullets; (R), (U), (F): educational material pages associated with the Remember, Use, Find levels of performance accordingly.

The approach of using multiple representations (knowledge modules) in the different instructional strategies that INSPIRE adopts, formulating alternative presentations of the educational material, alleviates the problem of rewriting the same content tailored to each learning style category. The different knowledge modules are either embedded in the page or appear as links in a specified order depending on the learning style of the learner. Thus, through the adaptive presentation technique, the same knowledge modules can be reused in different instructional strategies.

Adaptive Navigation Support. The system supports learner's navigation and orientation in the lesson contents by annotating the links that appear in the Navigation Area. Additional information is provided to the learner through the 
use of icons next to the names of concepts and the educational material. Different icons are used to distinguish between the outcome and the prerequisite concepts, as well as the educational material provided for each level of performance (notice in Fig.4, the different icons in the Navigation Area). Especially on the outcome concepts, the filling of a measuring cup is used as a metaphor denoting learner's progress.

Furthermore, two state icons accompany the prerequisite concepts and the educational material of the outcomes reflecting the instructional decisions of the lesson generation process on the educational material that the learner should study next. Thus, colored icons accompany the links that lead to the material that the system proposes the learner to study next, while black and white icons appear next to the rest of the links (see in Fig.4 the icons in the Navigation Area - the colored ones are denoted by a bullet).

Finally, a history-based mechanism has been developed so that, as each page is visited, a check mark appears next to its link in the Navigation Area (see Fig. 4 - Navigation Area).

\section{Implementation Issues}

INSPIRE is currently used to support an introductory course on Computer Architecture. In particular, educational material referring to the learning goal "What is the role of cache memory and its basic operations" has been developed based on the chapter Computer Memory of the module "Computer Architecture" 5 developed at the Department of Informatics and Telecommunications of the University of Athens. The current implementation of the system is using an IIS web server running on Windows NT, which processes the requests made by the learners. The learner model and the educational metadata describing the educational material [1, are stored in a SQL Server database that communicates with the web server through use of the ActiveX Data Objects (ADO) technology. The education material itself is stored in the file system pages. We are making use of the Active Server Pages (ASP) technology developed by Microsoft, which allows the dynamic generation of HTML page, in order to implement the adaptive presentation technique.

\section{Conclusions and Further Research}

INSPIRE is an adaptive system that monitors learner's activity and dynamically adapts the generated lessons to accommodate diversity in learners' knowledge state and learning style. An experiment focusing on the evaluation of the instructional design of the system has been conducted with students of the Department of Informatics and Telecommunication, who attend the course on Computer Architecture, and with participants of a seminar on the "Usability of Educational Software". The initial reactions towards the system have been encouraging while students' comments inspired several improvements on system's interface. 
The system is both adaptive and adaptable, as it allows the learner to control system's adaptation. The learner model of the system provides a complete description of the current state of the learner; it is open to the learner to make changes, and, in this way, allows him/her to intervene in the lesson generation process, supporting "end-learner modifiability". Further processing of the information stored in the learner model can be exploited by: (i) the system for the learner diagnosis process, (ii) the learner in order to be informed on system's decisions and intervene accordingly, and (iii) the tutor for the evaluation of the provided material and for monitoring learners' progress and study attitude. From the various statistics stored in the learner model the tutor can have a quantitative estimation of the learners' preferences on the educational material, in the sense of the time they spent on it, their performance, their attitude while studying, their progress, their requests to the system for help on specific pages etc.

The knowledge level and the learning style of the learner are used for the appropriate selection of the lesson contents and the presentation of the educational material. The domain structure is comprised by independent elements, i.e. concepts, educational material modules. These elements are reused in different learning goals and support multiple instructional strategies so that the different learners' educational needs and preferences can be fulfilled. In the current implementation of the system, the preferences of each individual learner are approached through his/her learning style, which is recognized through the submission of the appropriate questionnaire or defined by the learner. Further research is on progress concerning the estimation of the way each learner uses the educational material in order to identify inconsistencies in the association of the learning style of the learner (which has been detected by the system or specified by the learner) with the different types of educational material. For example, it is expected that the Activist will spent most of his/her time on activities and exercises, while the Reflector on theory presentations and examples. The way a learner uses the educational material in conjunction with his/her progress is valuable information denoting how successful is the selection of particular type of educational material for the particular learner. Furthermore, this information can also be used for the dynamic adaptation of the instructional strategy adopted for presentation of the educational material during learner's interaction with the system.

Acknowledgement. This work was partially supported by the Greek General Secretariat for Research and Technology of the Greek Ministry of Industry under a PENED99 grant No 99ED234.

\section{References}

1. ARIADNE project. Available at http://ariadne.unil.ch

2. Brusilovsky, P.: Adaptive and Intelligent Technologies for Web-based Education. In: Rollinger, C., Peylo, C. (eds.) Künstliche Intelligenz, Special Issue on Intelligent Systems and Teleteaching (1999) 
3. Brusilovsky, P.: Methods and Techniques of Adaptive Hypermedia. Learner Modeling and Learner-Adapted Interaction, Vol. 6. Kluwer Academic Publ., Netherlands (1996)

4. Entwistle, N.J.: Styles of Learning and Teaching. David Fulton, London (1988)

5. Grigoriadou M., Papanikolaou K., Cotronis Y., Velentzas Ch., Filokyprou G.: Designing and Implementing a Web-based course. In: Proc. of Int. Conf. of Computer Based Learning In Science, Enschede, Netherlands (1999) H5

6. Groat, A., Musson, T.: Learning Styles: individualising computer-based learning envi-ronments. ALT - Journal 3 (2) (1995) 53-62

7. Hartley, R., Paiva, A., Self J.: Externalizing Learner Models. In: Greer, J. (ed.): Proc. of Int. Conf. on Artificial Intelligence in Education. AACE, Washington (1995) 509-516

8. Honey, P., Mumford, A.: The manual of Learning Styles. Peter Honey Maidenhead (1992)

9. Kolb, D. A.: Experiential learning. Englewood Cliffs, Prentice-Hall, NJ (1984)

10. Magoulas, G.D., Papanikolaou, K.A., Grigoriadou, M.: Neuro-fuzzy Synergism for Planning the Content in a Web-based Course. Informatica 25 (1) (2001) 39-48

11. Marcke, V.: A Generic Task Model for Instruction. Instructional models for Computer-based Learning Environments. Nato ASI Series F, Vol. 104. SpringerVerlag, Berlin Heidelberg New York (1992)

12. McLoughlin, C.: The implications of the research literature on learning styles for the design of instructional material. Australian J. of Educational Technology 15 (3) (1999)

13. Merril, M.D.: Component Display Theory. In: Reigeluth, C.M. (ed.): Instructional design theories and models: An overview of their current status. Lawrence Elrbaum Association, Hillsdale NJ (1983)

14. Papanikolaou, K.A., Grigoriadou, M., Kornilakis, H., Magoulas, G.D.: Towards New Forms of Knowledge Communication: An INtelligent System for Personalised Instruction in a Remote Environment (INSPIRE) (Submitted)

15. Papanikolaou, K.A., Magoulas, G.D., Grigoriadou, M.: A Connectionist Approach for Supporting Personalized Learning in a Web-based Learning Environment. In: Brusilovsky, P., Stock, O., Strapparava, C. (eds.): Adaptive Hypermedia and Adaptive Web-based Systems. Lecture Notes in Computer Science, Vol. 1892. SpringerVerlag, Berlin Heidel-berg New York (2000) 189-201

16. Stoyanov, S., Aroyo, L., Kommers, P.: Intelligent Agents Instructional Design Tools for a Hypermedia Design Course. In: Lajoie, S.P., Vivet, M. (eds.): Artificial Intelligence in Education. IOS Press (1999)

17. Vosniadou, S.: Towards a revised cognitive psychology for new advances in learning and instruction. Learning and Instruction 6(2) (1996) 95-109 